\title{
Simultaneous small-angle X-ray scattering/wide-angle X-ray diffraction study of the microdomain structure of polyurethane elastomers during mechanical deformation
}

\author{
Ken Kojio $^{1}$, Keisuke Matsuo ${ }^{1}$, Suguru Motokucho ${ }^{1}$, Kohji Yoshinaga ${ }^{1}$, Yoshiki Shimodaira ${ }^{2}$ \\ and Kazuya Kimura ${ }^{2}$
}

Polyurethane elastomers (PUEs) were prepared with poly(oxytetramethylene) glycol ( $M_{\mathrm{n}}=2000$ ), 4,4'-diphenylmethane diisocyanate, 1,4-butanediol (BD) and 1,1,1-trimethylol propane (TMP) by a prepolymer method. To evaluate the effect of curing temperature and the ratio of curing and crosslinking agents $((B D / T M P)=(10 / 0$ and $8 / 2)(w t / w t))$ on deformation behavior, four different samples were prepared. In the small-angle X-ray scattering (SAXS) profile for the PUEs prepared at $120^{\circ} \mathrm{C}$, a four-point pattern was observed with the preferred tilt being produced by local torques exerted within the strained soft segments from the initial deformation. At near failure strains, strong meridional scattering appeared and the four-point pattern disappeared. In contrast, the PUEs at $80^{\circ} \mathrm{C}$ produced meridional scattering through the deformation. As the microdomain structure of the PUEs prepared at 120 and $80^{\circ} \mathrm{C}$ initially possessed cylinder- and sphere-like structures, respectively, the cylinder-like structure might have produced the four-point pattern. Obvious changes in interdomain spacing of PUEs at $120{ }^{\circ} \mathrm{C}$ during the deformation process were observed in comparison with the spacing at $80^{\circ} \mathrm{C}$. This was due mainly to the formation of a well-developed, networked, cylinder-like microdomain structure. Strain-induced crystallization of the soft-segment chains evaluated by wide-angle X-ray diffraction results was also consistent with the results from SAXS.

Polymer Journal (2011) 43, 692-699; doi:10.1038/pj.2011.48; published online 8 June 2011

Keywords: mechanical deformation; microdomain structure; polyurethane elastomer

\section{INTRODUCTION}

Polyurethane (PU) is an intriguing polymer material because various types of final PUs can be obtained by mixing only two raw materials. ${ }^{1,2}$ PUs could be used as foam, coating, adhesive, sealant, elastomer, fiber, sole and biomaterial, ${ }^{3-5}$ which suggests that $\mathrm{PU}$ is a polymer with the widest industrial applications in the world. The key factor for the broad usage of PUs is likely the formation of hydrogen bonds between strong polar urethane groups. However, there are a number of open questions on the structure-property relationship of PUs.

The PU molecules in PU elastomers (PUEs) need to form a structure with separated microphases consisted of the domains with hard segments and a matrix with soft segments. The microphaseseparated structure is influenced by many factors, such as the chemical structure and weight fraction of both the hard and the soft components, the molecular weights, the polydispersity of the soft segment and preparation conditions including thermal history. ${ }^{1-23}$ The various properties including mechanical property of the PUEs would be affected by the microphase-separated structure not only in the initial state but also in the elongated one.

The deformation behavior of PUE has been investigated extensively using a variety of experimental techniques. ${ }^{24-28}$ However, the deformation behavior of the PUE is still not well understood because a tremendous number of factors need to be considered to control the original phase structure. Systematic sample preparation and mechanical measurements for the samples would give us a general interpretation on this topic.

In this study, we prepared four PUEs at different temperatures and with different ratios of curing and crosslinking agents. Then, smallangle X-ray scattering (SAXS)/wide-angle X-ray diffraction (WAXD) measurements were simultaneously performed on the samples with and without deformation. The relationship between the initial microphase-separated structure and the deformation behavior was investigated. The results were important in designing the molecules to control the various properties of the PUEs.

${ }^{1}$ Division of Chemistry and Materials Science, Graduate School of Engineering, Nagasaki University, Nagasaki, Japan and ${ }^{2}$ Corporate R\&D Center, Showa Denko K. K., Chiba, Japan

Correspondence: Professor K Kojio, Division of Chemistry and Materials Science, Graduate School of Engineering, Nagasaki University, 1-14 Bunkyo-machi, Nagasaki 852-8521, Japan. 


\section{EXPERIMENTAL PROCEDURE}

\section{Materials}

Poly(oxytetramethylene) glycol (PTMG: $M_{\mathbf{n}}=2000$, Asahikasei Chemicals, Tokyo, Japan), 4,4'-diphenylmethane diisocyanate (MDI, Nippon Polyurethane Industry, Tokyo, Japan) and 1,4-butanediol (BD, Wako Chemicals, Kanagawa, Japan) were used as the polymer glycol, diisocyanate and chain extender, respectively. Purity of MDI was $>99.5 \%$. BD was refluxed with calcium hydride to remove water and subsequently distilled for purification. MDI was used without further purification.

\section{Synthesis of PUEs}

The PUEs were synthesized by a bulk prepolymer method. The PTMG was dried with dry nitrogen under reduced pressure. Prepolymers were prepared from PTMG and MDI with a ratio of $K=[\mathrm{NCO}] /[\mathrm{OH}]=3.3$ at $80^{\circ} \mathrm{C}$ for $3 \mathrm{~h}$ under a nitrogen atmosphere. The extent of the reaction was determined by an amine equivalent method. After the reaction was finished, the prepolymer was placed in vacuum to remove the air inside. The prepolymer and crosslinking agent were mixed well at a ratio of $[\mathrm{NCO}]_{\mathrm{pre}} /[\mathrm{OH}]=1.05$, where $[\mathrm{NCO}]_{\text {pre }}$ is the concentration of $\mathrm{NCO}$ groups in a prepolymer. After the viscous product was mixed for $90 \mathrm{~s}$, it was poured into a mold constructed using a $1 \mathrm{~mm}$ thick spacer and two aluminum plates heated to 80 and $120^{\circ} \mathrm{C}$. To control the chemical crosslinking density, BD-to-trimethylol propane (TMP) ratios (wt/ wt) of $(10 / 0)$ and $(8 / 2)$ were investigated. Because BD and TMP are difunctional and trifunctional molecules, respectively, PUEs with a ratio of $(10 / 0)$ and $(8 / 2)$ have linear and partially crosslinked structures, respectively. The PUEs were demolded after a $2 \mathrm{~h}$ curing, followed by post-curing at $80^{\circ} \mathrm{C}$ for $24 \mathrm{~h}$ in air. Four PUEs were synthesized using various curing temperatures and TMP contents, and their nomenclature denoted the $\mathrm{BD} / \mathrm{TMP}$ ratio and curing temperature.

\section{SAXS and WAXD measurements}

SAXS and WAXD data were acquired at the BL03XU ${ }^{29}$ beamline in the SPring- 8 facility in Japan. The typical beam flux was $2 \times 10^{12}$ photons $\mathrm{s}^{-1}$, and the size of beam at the detector was $140 \times 80 \mu \mathrm{m}^{2}$. The X-ray with a $0.1 \mathrm{~nm}$ wavelength was monochromatized from the bending magnet using a monochromator. For SAXS measurements, a CCD detector with a pixel size of $63 \mu \mathrm{m}$ per pixel and a total size of $1344 \times 1024$ pixels was used to measure the scattered radiation. The detector was placed behind a vacuum path, and the camera length was $1.7 \mathrm{~m}$. For WAXD measurements, a flat panel detector was used, and the camera length was $63 \mathrm{~mm}$.

A home-built stretcher was used to elongate the $\sim 0.2$-mm thick samples. Scattering patterns were collected for $\sim 1 \mathrm{~s}$. Data were reduced from the twodimensional (2D) format to one-dimensional (1D) by integrating with FIT2D (Ver. 12.077, Andy Hammersley). Both sets of data were expressed in terms of the wave vector, $q$, where $q=4 \pi \sin \theta / \lambda$.

\section{RESULTS AND DISCUSSION}

\section{Microdomain structure of PUEs}

The PUEs synthesized in this study appeared opaque mainly because of the crystallites of the hard segments. The microphase-separated structure of the PUEs is strongly dependent on the BD/TMP ratio and the curing temperature. ${ }^{1-3}$ First, we investigated the phase structure of four PUEs without deformation being denoted the following sample IDs: $10 / 0-80,10 / 0-120,8 / 2-80$ and 8/2-120.

Figure 1 shows the (a) SAXS and (b) WAXD profiles for four samples measured at a strain of zero. In the SAXS profile, scatterings due to hard and soft segments were observed. Because the spacing obtained from these peaks was $\sim 20 \mathrm{~nm}$, it is conceivable that the scatterings could be assigned to the interdomain spacing of the hardsegment domains. Peak positions for the $10 / 0-120$ and $8 / 2-120$ samples were observed at a lower $q$ range than those for the $10 / 0-80$ and 8/2-80 samples, indicating that the interdomain spacings of the $10 / 0-120$ and 8/2-120 samples were larger than those of the $10 / 0-80$ and $8 / 2-80$ samples. The magnitude of the interdomain spacings was $19,24,19$ and $28 \mathrm{~nm}$ for samples 10/0-80, 10/0-120, 8/2-80 and
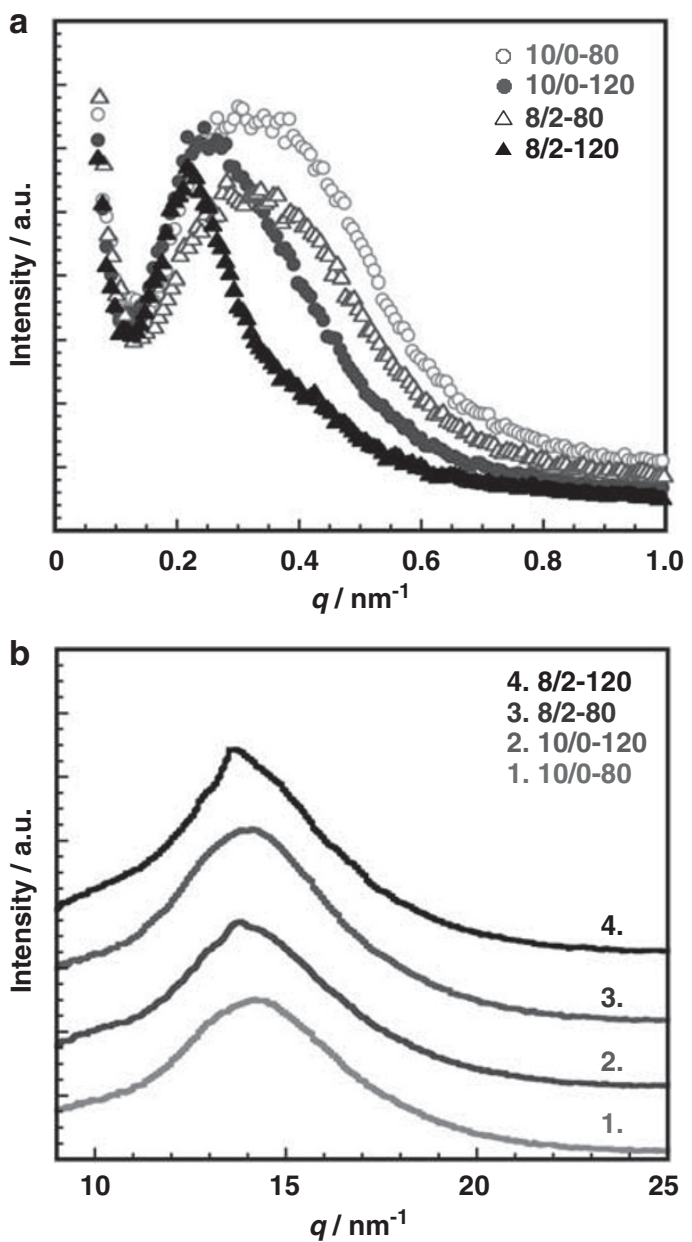

Figure 1 (a) Small-angle X-ray scattering and (b) wide-angle X-ray diffraction profiles for four polyurethane elastomer samples measured at zero strain. A full color version of this figure is available at Polymer Journal online.

$8 / 2-120$, respectively. The peak widths for $10 / 0-120$ and $8 / 2-120$ were much sharper than those for $10 / 0-80$ and $8 / 2-80$, as clearly observed in Figure 1a. The crystallized hard-segment chains were composed of MDI and BD with melting points around $200{ }^{\circ} \mathrm{C} ., 8,13,14$ Therefore, curing at a higher temperature seemed to bring the crystallites closer to the equilibrium state. That is, the crystallites in the equilibrium state possessed larger sizes with small size distributions. The effect of the trifunctional TMP content was evidenced in samples $8 / 2-80$ and $8 / 2-120$, which exhibited peaks at a lower $q$ range with sharper widths. Generally speaking, chain extender, TMP, which has three hydroxyl groups, give a disordered crystal structure of the hardsegment chains. However, the results obtained in this study showed an opposite trend. The long -(MDI-BD $)_{n}$ - chains might not have enough freedom to form the well-organized crystallites because of their chain rigidity and strong interaction among molecular chains. ${ }^{30}$ Therefore, incorporating TMP may provide the hard-segment chains with the necessary freedom and the proper length to form wellordered hard-segment domains.

In the WAXD profiles, the 10/0-80 and 8/2-80 PUEs showed only an amorphous halo at $14.0 \mathrm{~nm}^{-1}$. In contrast, the $10 / 0-120$ and $8 / 2-120$ samples exhibited weak peaks and shoulders corresponding to the crystallized hard-segment domains at 13.8 and $15.8 \mathrm{~nm}^{-1}$. $^{31}$ Thus, the hard-segment chains in the PUE prepared at $120^{\circ} \mathrm{C}$ were well crystallized in comparison with those at $80^{\circ} \mathrm{C}$. 

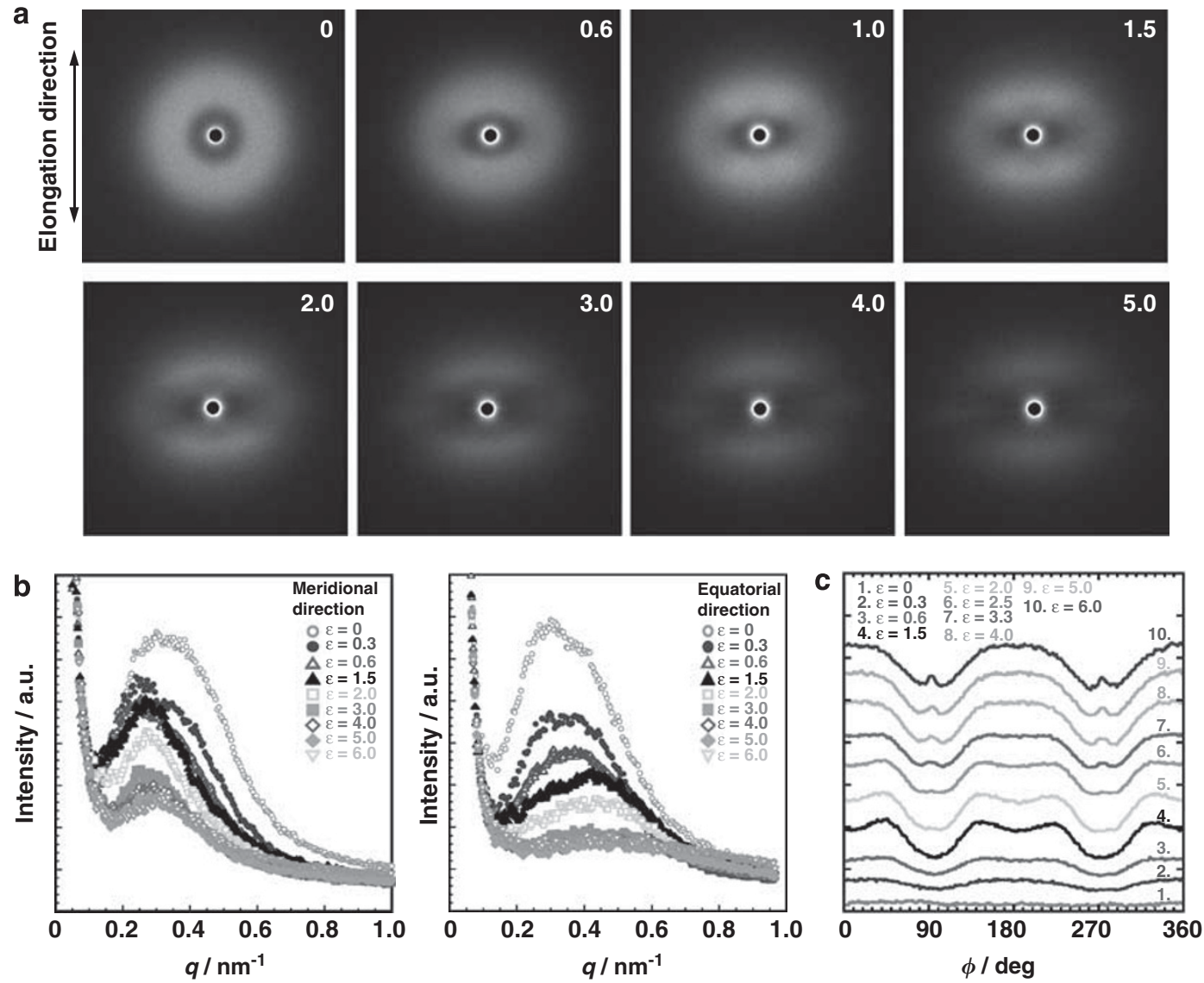

Figure 2 (a) The evolution of two-dimensional (2D)-small-angle X-ray scattering (SAXS) patterns for the 10/0-80 polyurethane elastomer sample as a function of strain during elongation. (b) The corresponding one-dimensional-SAXS linear intensity profiles for the meridional and equatorial regions at various strains. (c) The azimuthal angle profiles of the $2 \mathrm{D}$ pattern at $q=0.4 \mathrm{~nm}^{-1}$. A full color version of this figure is available at Polymer Journal online.

We directly observed the microdomain structure of these PUEs using atomic force microscopy (Kojio et al. (unpublished results)). As a result, it was determined that the structure of the $10 / 0-80$ and $8 / 2-80$ PUEs consisted of isolated hard-segment domains surrounded by a soft-segment matrix. The sizes of the hard-segment domains were $10-20 \mathrm{~nm}$. However, the structures of the $10 / 0-120$ and $8 / 2-120$ samples were formed by two characteristic regions. One region contained isolated hard-segment domains with a surrounding softsegment matrix, and the domain sizes were $10-20 \mathrm{~nm}$ in diameter. The other region contained branch-like hard-segment domains with diameters of $20-30 \mathrm{~nm}$. In summary, the $10 / 0-80$ and $8 / 2-80$ PUEs possessed a sphere-type microdomain structure, whereas the $10 / 0-120$ and 8/2-120 PUEs had a cylinder-like structure.

\section{Microdomain structure change during mechanical deformation}

10/0-80 PUE. Figure 2a shows the evolution of the 2D-SAXS patterns for the 10/0-80 PUE as a function of strain during elongation. Figure $2 \mathrm{~b}$ depicts the corresponding 1D-SAXS intensity profiles for the meridional and equatorial regions at various strains. Figure $2 \mathrm{c}$ shows the azimuthal angle profiles of the $2 \mathrm{D}$ pattern at $q=0.4 \mathrm{~nm}^{-1}$. For each profile, the mean value of $q$ was $0.4 \mathrm{~nm}^{-1}$ with a range was $0.25 \mathrm{~nm}^{-1}$. The stretching direction corresponded to the meridional axis in the SAXS patterns. In the early stages of deformation, the circular 2D-SAXS patterns in Figure 2a became elliptical with the small radius aligned with the meridional axis, indicating that the interdomain spacings increased along the tensile axis and decreased in the direction transverse to the tensile axis. As shown in Figure 2b, in the early stages of deformation, the intensity at the meridional axis (which was at the azimuthal angle with $\varphi=0^{\circ}$ and $180^{\circ}$ ) was stronger than that at the equatorial axis $\left(\varphi=90^{\circ}\right.$ and $\left.270^{\circ}\right)$. As strain increased, weak fourpoint pattern appeared as shown in Figure $2 b$. Strong meridional scattering appeared shortly after, coupled with disappearance of the four-point pattern. Also of note was the equatorial streaking that appeared at a strain of 3.3. The intensity of the streaking increased with strain (Figure 2c).

Figures $3 \mathrm{a}$ and $\mathrm{b}$ show the 2D-WAXD patterns and the corresponding 1D-WAXD intensity profile, respectively, for the 10/0-80 PUE sample as a function of strain during elongation. In Figures $3 a$ and $b$, an amorphous halo was observed at zero strain. However, two high intensities of diffraction peaks were observed on the equatorial axis at a strain of 5.0. The two peaks observed at $q=14.2$ and $16.8 \mathrm{~nm}^{-1}$ corresponded well with the (020) and (110) of the crystallized PTMG chain. ${ }^{32}$ Therefore, it is conceivable that the strain-induced crystallization occurred with increasing strain. Even though the structure change in the hard-segment domains occurred at a strain of 0.3 as shown in Figure 2, the strain-induced crystallization of the softsegment chains occurred at a much greater strain $(\sim 3.0)$. Quantitative discussion will be given in the later section.

10/0-120 PUE. Figure 4a shows the evolution of the 2D-SAXS patterns for the 10/0-120 PUE sample as a function of strain during elongation. Figure $4 \mathrm{~b}$ depicts the corresponding 1D-SAXS intensity 

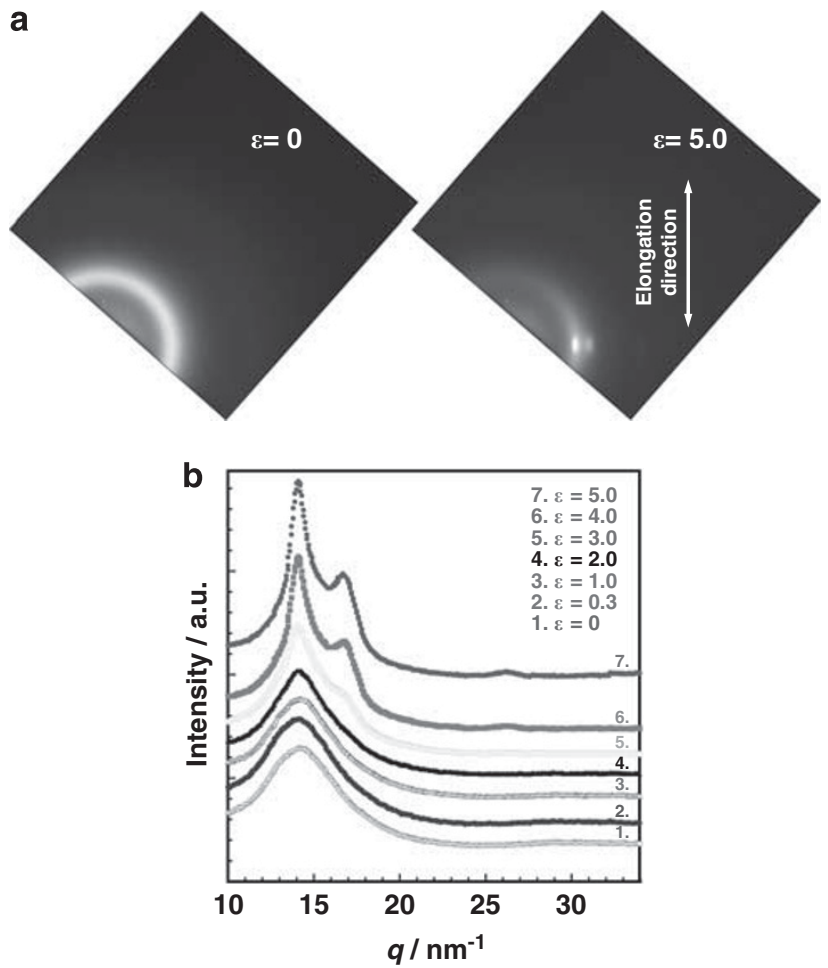

profiles for the meridional and equatorial regions at various strains. Figure $4 \mathrm{c}$ shows the azimuthal angle profiles of the $2 \mathrm{D}$ pattern at $q=0.4 \mathrm{~nm}^{-1}$. For each profile, the mean value of $q$ was $0.4 \mathrm{~nm}^{-1}$, and the range was $0.25 \mathrm{~nm}^{-1}$. The initially isotropic scattering pattern quickly displayed anisotropy in the form of four-point scattering at a strain of 0.3 . As the intensity of the four-point pattern increased with strain, meridional scattering began to appear and coincided with the beginning of hard segment reorganization. As strain increased to 3.6, the initial four-point scattering characteristic of tilted cylinder almost completely disappeared, indicating that the majority of the hardsegment domains had undergone reorganization. Also of note was the equatorial streaking that appeared at near failure strains, which was characteristic of the beginning of oriented nanofibril formation. Figure $4 \mathrm{~b}$ shows the 1D-SAXS profiles along the equatorial and meridional directions, which could be used to discuss the orientation of the hard-segment domains. Whereas the equatorial intensity decreased monotonically with increasing strain, the meridional intensity started decreasing after a strain of $\sim 1.5$ and dropped off noticeably, suggesting the beginning of a dramatic breakdown in the hard-segment domains.

Figure 3 (a) The evolution of two-dimensional-wide-angle X-ray diffraction (WAXD) patterns for the 10/0-80 polyurethane elastomer sample as a function of strain during elongation. (b) The one-dimensional-WAXD intensity profiles for the equatorial region at various strains. a
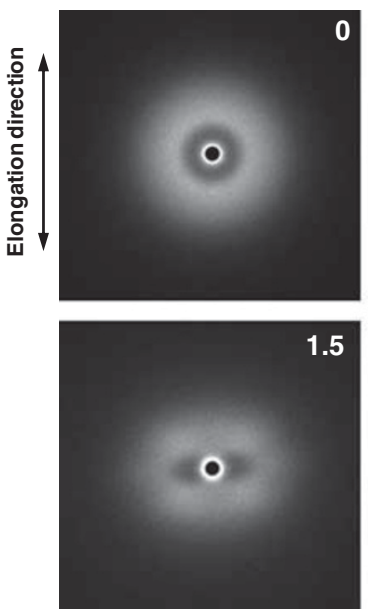

b

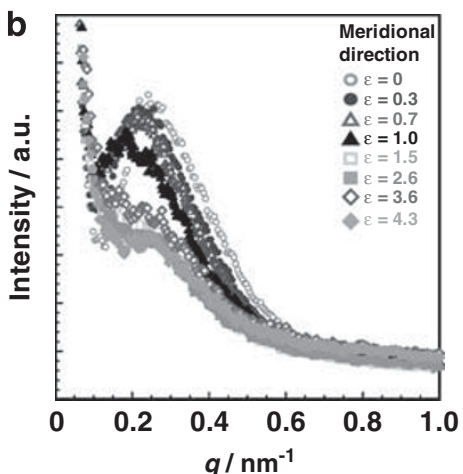

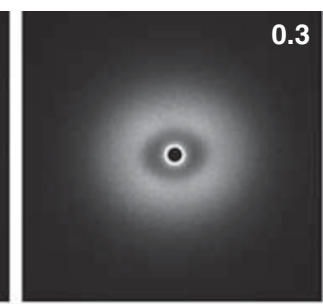

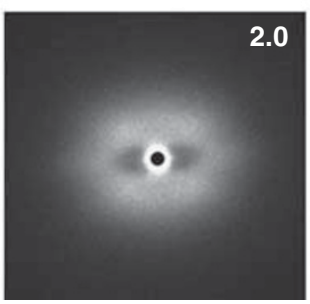

2.0
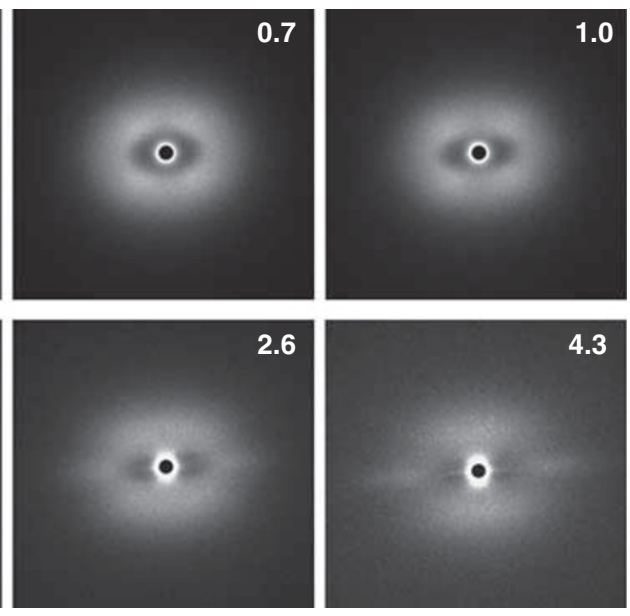

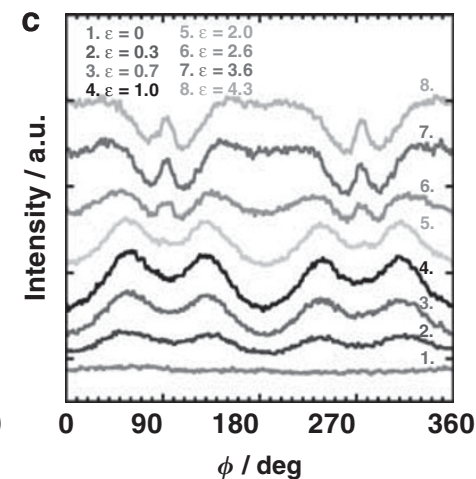

Figure 4 (a) The evolution of two-dimensional (2D)-small-angle X-ray scattering (SAXS) patterns for the 10/0-120 polyurethane elastomer sample as a function of strain during elongation. (b) The corresponding one-dimensional-SAXS linear intensity profiles for the meridional and equatorial regions at various strains. (c) The azimuthal angle profiles of the $2 \mathrm{D}$ pattern at $q=0.4 \mathrm{~nm}^{-1}$. A full color version of this figure is available at Polymer Journal online. 


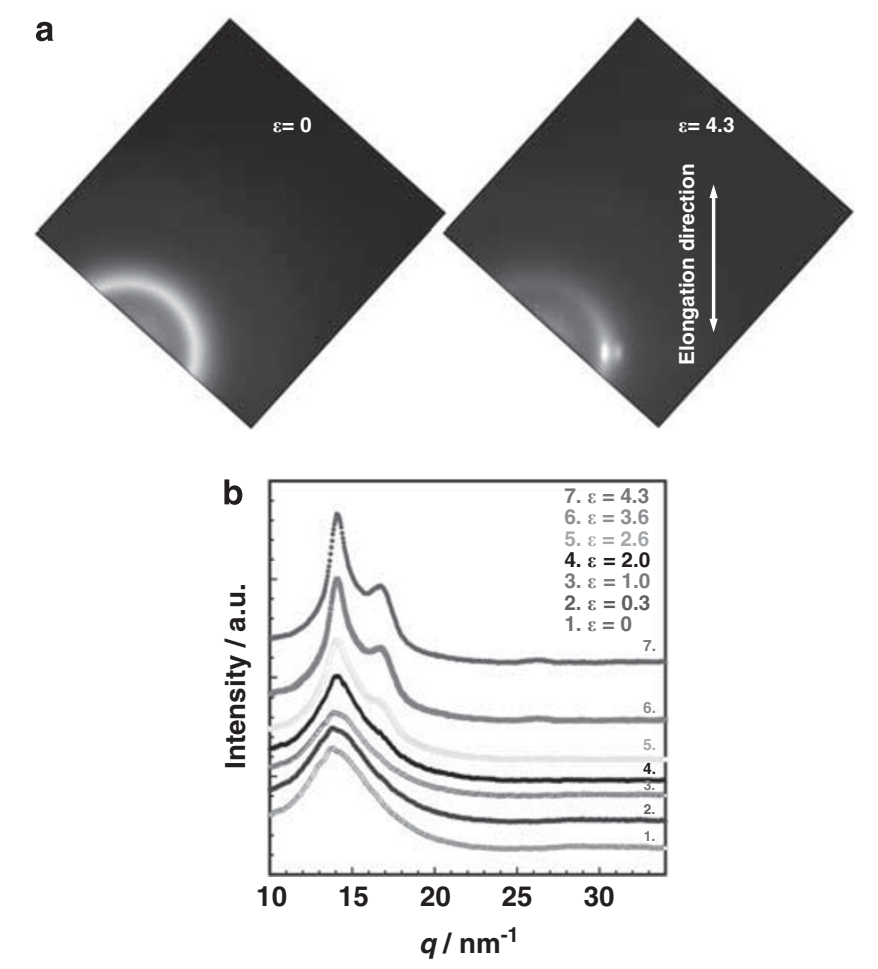

Figures $5 \mathrm{a}$ and $\mathrm{b}$ show the $2 \mathrm{D}$-WAXD patterns and the corresponding 1D-WAXD intensity profiles, respectively, for the 10/0-120 PUE sample as a function of strain during elongation. In Figures $5 \mathrm{a}$ and $\mathrm{b}$, a tiny peak was observed at $q=13.8 \mathrm{~nm}^{-1}$ even at zero strain, which could be assigned to crystallized hard-segment chains. As the strain increased to more than 2.0, two peaks were observed on the equatorial axis, which were also observed in the 10/0-80 sample (Figure 3). Therefore, it is likely that the strain-induced crystallization of the soft segment occurred.

8/2-80 PUE. Figure 6 a shows the evolution of the 2D-SAXS patterns for the 8/2-80 PUE as a function of strain during elongation. Figure $6 \mathrm{~b}$ depicts the corresponding 1D-SAXS intensity profiles for the meridional and equatorial regions at various strains. Figure $6 c$ shows the azimuthal angle profiles of the $2 \mathrm{D}$ pattern at $q=0.4 \mathrm{~nm}^{-1}$. For each profile, the mean value of $q$ was $0.4 \mathrm{~nm}^{-1}$, and the range was $0.25 \mathrm{~nm}^{-1}$. The initial appearance of the meridional scattering and weak four-point scattering during the elongation process for the 8/2-80 PUE sample were similar to the 10/0-80 PUE sample. The

Figure 5 (a) The evolution of two-dimensional-wide-angle X-ray diffraction (WAXD) patterns for the 10/0-120 polyurethane elastomer sample as a function of strain during elongation. (b) The one-dimensional-WAXD intensity profiles for the equatorial region at various strains. A full color version of this figure is available at Polymer Journal online.
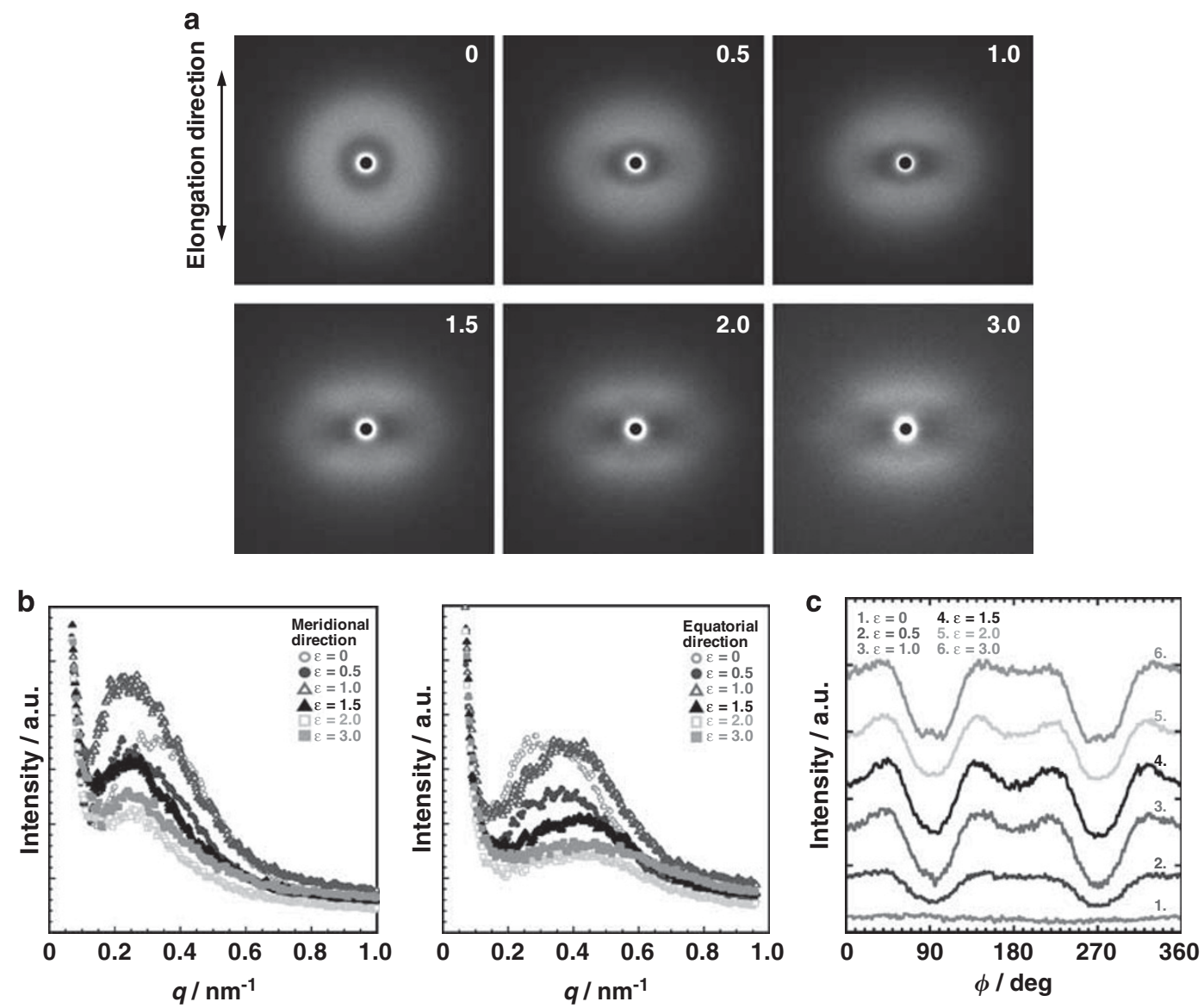

Figure 6 (a) The evolution of two-dimensional (2D)-small-angle X-ray scattering (SAXS) patterns for the 8/2-80 polyurethane elastomer as a function of strain during elongation. (b) The corresponding one-dimensional-SAXS linear intensity profiles for the meridional and equatorial regions at various strains. (c) The azimuthal angle profiles of the 2D pattern at $q=0.4 \mathrm{~nm}^{-1}$. A full color version of this figure is available at Polymer Journal online. 
difference between the two samples was that no obvious streaking was observed for the 8/2-80 PUE sample. This difference could be associated with the existence of trifunctional TMP in the 8/2-80 PUE sample.

Figures $7 \mathrm{a}$ and $\mathrm{b}$ show the 2D-WAXD patterns and the corresponding 1D-WAXD intensity profiles, respectively, for the 8/2-80 PUE as a function of strain during elongation. In Figures $7 a$ and $b$, an amorphous halo was observed at a zero strain. Weak peaks at $q=14.2$ and $16.8 \mathrm{~nm}^{-1}$ were observed shortly after at a strain of 3.0. The peak intensity was clearly weaker than that in the $10 / 0-80$ and 10/0-120 samples. This result was because the trifunctional TMP suppressed the crystallization of not only hard-segment chains at the initial state but also the soft-segment chains during deformation.

8/2-120 PUE. Figure 8a shows the evolution of the 2D-SAXS patterns for the 8/2-120 PUE as a function of strain during elongation.
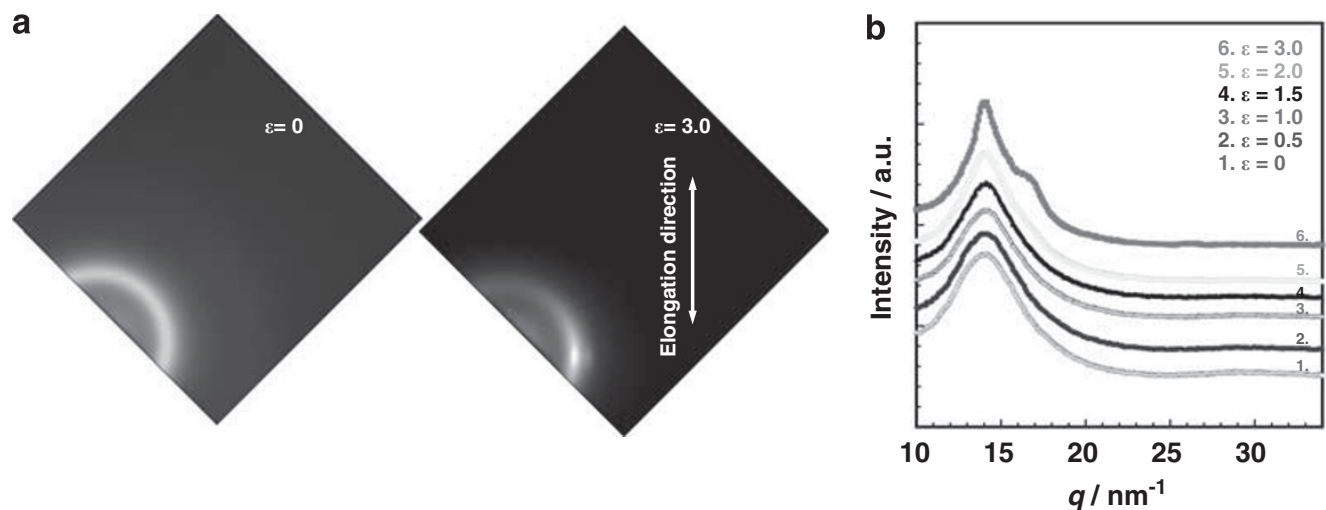

Figure 7 (a) The evolution of two-dimensional-wide-angle X-ray diffraction (WAXD) patterns for the 8/2-80 polyurethane elastomer sample as a function of strain during elongation. (b) The one-dimensional-WAXD intensity profiles for the equatorial region at various strains. A full color version of this figure is available at Polymer Journal online.
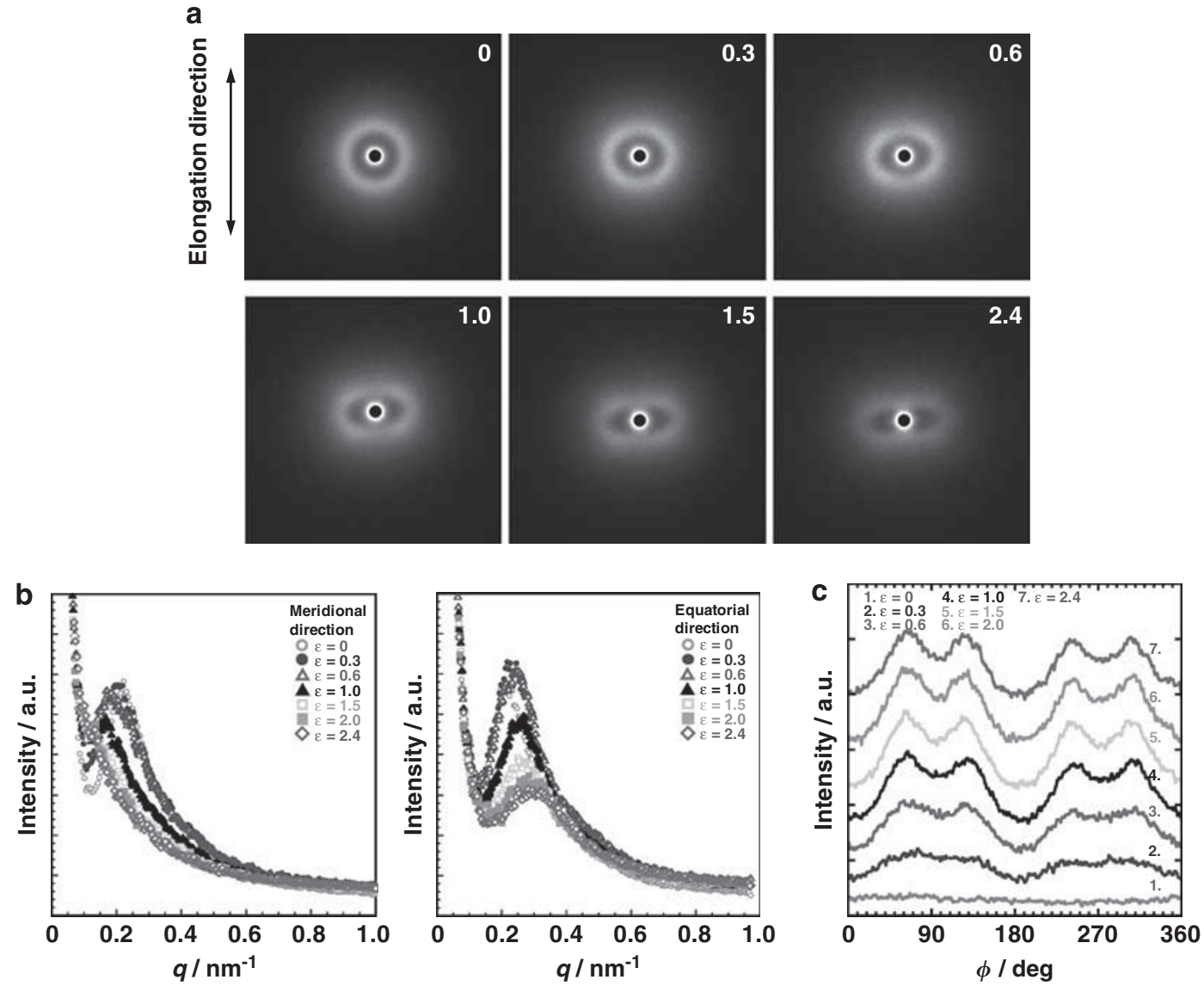

Figure 8 (a) The evolution of two-dimensional (2D)-small-angle X-ray scattering (SAXS) patterns for the 8/2-120 polyurethane elastomer sample as a function of strain during elongation. (b) The corresponding one-dimensional-SAXS linear intensity profiles for the meridional and equatorial regions at various strains. (c) The azimuthal angle profiles of the 2D pattern at $q=0.4 \mathrm{~nm}^{-1}$. A full color version of this figure is available at Polymer Journal online. 


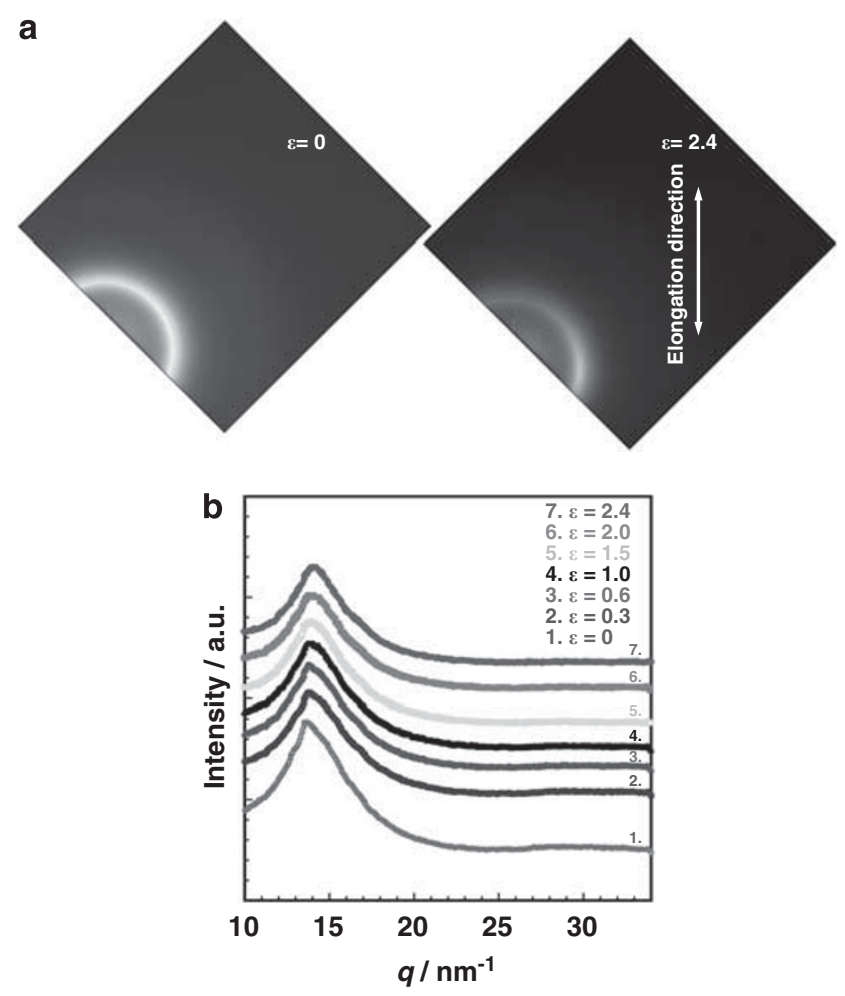

Figure 9 (a) The evolution of two-dimensional-wide-angle $X$-ray diffraction (WAXD) patterns for the 8/2-120 polyurethane elastomer sample as a function of strain during elongation. (b) The one-dimensional-WAXD intensity profiles for the equatorial region at various strains. A full color version of this figure is available at Polymer Journal online.

Figure $8 \mathrm{~b}$ depicts the corresponding 1D-SAXS intensity profiles for the meridional and equatorial regions at various strains. Figure $8 \mathrm{c}$ shows the azimuthal angle profiles of the $2 \mathrm{D}$ pattern at $q=0.4 \mathrm{~nm}^{-1}$. For each profile, the mean value of $q$ was $0.4 \mathrm{~nm}^{-1}$, and the range was $0.25 \mathrm{~nm}^{-1}$. The initial appearance of the meridional scattering and weak four-point scattering during the elongation process for the $8 / 2$ 120 PUE sample were similar to the 10/0-120 PUE sample. However, no streaking was observed for the 8/2-120 PUE sample even at larger strains because of the existence of trifunctional TMP. In addition, the scattering peak along the meridional axis nearly disappeared at strains $>1.6$, as shown in Figure $8 \mathrm{~b}$. This phenomenon could be due to the breakdown of hard-segment domains and the increasing interdomain spacing with elongation. This result could be attributed to the formation of well-organized hard-segment domains and the existence of chemical crosslinking points.

Figures $9 \mathrm{a}$ and $\mathrm{b}$ shows the 2D-WAXD patterns and the corresponding 1D-WAXD intensity profiles, respectively, for the 10/0-120 PUE as a function of strain during elongation. In Figures $9 a$ and $b$, a tiny peak was observed at $q=13.8 \mathrm{~nm}^{-1}$, identical to the $10 / 0-120$ sample in the initial state. In the case of the 8/2-120 PUE sample, the elongation at break was small because of the existence of welldeveloped hard-segment domains and chemical crosslinking points. Thus, obvious diffraction peaks from strain-induced and crystallized PTMG chains were not observed.

Interdomain spacing of hard-segment domains and crystallinity of soft-segment chains

Figure 10 shows the strain dependence of the interdomain spacing obtained from SAXS measurements for the four PUEs prepared in this

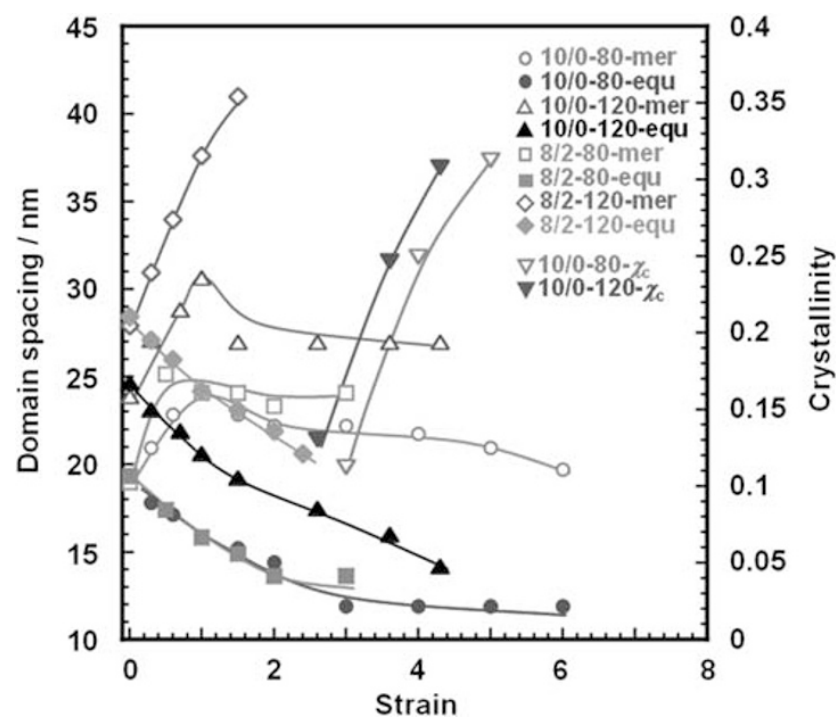

Figure 10 Strain dependence of interdomain spacing for the four polyurethane elastomers prepared in this study. The crystallinity of the hardsegment chains was also plotted. A full color version of this figure is available at Polymer Journal online.

study. The crystallinity of the soft-segment chains during the straininduced crystallization process obtained from WAXD measurements was also plotted. The initial interdomain spacings of the PUEs prepared at $120^{\circ} \mathrm{C}$ were larger than those of the PUEs prepared at $80^{\circ} \mathrm{C}$. As discussed previously, curing at high temperatures induces the formation of well-organized hard-segment domains. As a result, the 10/0-120 and 8/2-120 samples exhibited larger interdomain spacings with narrower distribution in comparison with the $10 / 0-80$ and 8/2-80 samples.

For all four PUEs, the interdomain spacings along the meridional increased with strain, whereas the spacings along the equatorial axes decreased with strain. For the 10/0-80 sample, the interdomain spacing along the meridional axis increased with strain to a constant value, whereas the spacing along the equatorial axis gradually decreased. A similar trend was observed for the 8/2-80 sample. However, the interdomain spacing along the meridional and equatorial axes steeply increased and decreased as strain increased for the PUEs prepared at $120^{\circ} \mathrm{C}$. In other words, there was significant difference in the hard-segment domain structure between the meridional and equatorial axes during the elongation process, which was because the applied strain quickly propagated to the hard-segment domains due to their well-organized and cylinder-like structure. For the 10/0-80 and 10/0-120 samples, the interdomain spacing in the higher strain region tended to be constant. These results indicate that the hard-segment domains in the two samples reorganized because of the lack of chemical crosslinking points. The fact that streaking was observed for these two samples (Figures $2 \mathrm{c}$ and $4 \mathrm{c}$ ) supported this trend. The physical crosslinking points in the PUEs could reorganize easily because the hydrogen bonds could break and form quickly during the deformation process. The crystallinity $\left(\chi_{c}\right)$ of the softsegment chains could be obtained only at a strain $>3.0$. The magnitude of $\chi_{\mathrm{c}}$ of the 10/0-120 sample was larger than that of the 10/0-80 sample, which was rather consistent with the SAXS result. That is, the applied strain propagated easily in the 10/0-120 sample because of the cylinder-like microdomain structure with well-ordered crystallites. The initial microdomain structure was closely related to the mechanical deformation behavior. 


\section{CONCLUSIONS}

We investigated the relationship between the microdomain structures of the PUEs prepared with different curing temperatures and curing/ crosslinking agents in the initial state and during the deformation process. SAXS measurements revealed that the $10 / 0-80$ and $8 / 2-80$ PUEs possessed a sphere-type microdomain structure, whereas the 10/0-120 and 8/2-120 PUEs had a cylinder-like structure in the initial state. The hard-segment chains in the 10/0-80 and 8/2-80 PUEs formed a layer structure normal to the deformation direction, whereas those in the 10/0-120 and 8/2-120 PUEs formed a tilted cylinder structure following the formation of a layer structure. The occurrences of these phenomena were dependent on the existence of the cylinderlike structure. The PUEs without TMP (10/0-80 and 10/0-120) exhibited streaking on the equatorial axis at larger strains because of the formation of oriented nanofibrils. These results could be useful for the molecular design of PUEs with high functionality and performance.

\section{ACKNOWLEDGEMENTS}

We thank Dr Mutsuhisa Furukawa and Mr Shun Matsumura for synthesizing the samples used in this study. We also gratefully acknowledge Dr Hiroyasu Masunaga (Japan Synchrotron Radiation Research Institute (JASRI)), Dr Hiroki Ogawa (JASRI) and Dr Takumi Takano (JASRI) for their assistance on the SAXS/WAXD measurements. The synchrotron radiation experiments were performed at the SPring-8 facility with the approval of the Japan Synchrotron Radiation Research Institute (JASRI; Proposal No. 2010A7210).

1 Ng, H. N., Allegrezza, A. E., Seymour, R. W. \& Cooper, S. L. Effect of segment size and polydispersity on the properties of polyurethane block polymers. Polymer 14, 255-261 (1973).

2 Petrovic, Z. S. \& Ferguson, J. Polyurethane elastomers. Prog. Polym. Sci. 16, 695-836 (1991).

3 Koberstein, J. T. \& Russell, T. P. Simultaneous SAXS-DSC study of multiple endothermic behavior in polyether-based polyurethane block copolymers. Macromolecules 19, 714-720 (1986).

4 Cunningham, A. Modulus anisotropy of low-density cellular plastics: an aggregate model. Polymer 22, 882-885 (1981).

5 Takahara, A., Takahashi, K. \& Kajiyama, T. Effect of polyurethane surface chemistry on its lipid sorption behavior. J. Biomater. Sci. Polym. Ed. 5, 183-196 (1993).

6 Silver, J. H., Karayianni, E. \& Cooper, S. L. Effect of polyol hydrophilicity on the structure and anticoagulant activity of water-soluble sulfonated polyurethanes. J. Coll. Inter. Sci. 178, 219-232 (1996).

7 Furukawa, M., Komiyama, M. \& Yokoyama, T. Characterization of polyurethane network elastomers. Angew. Makro. Chem. 240, 205-211 (1996).

8 Furukawa, M., Hamada, Y. \& Kojio, K. Aggregation structure and mechanical properties of functionally graded polyurethane elastomers. J. Polym. Sci. Polym. Phys. 41, 2355-2364 (2003).

9 Kojio, K., Fukumaru, T. \& Furukawa, M. Highly softened polyurethane elastomer synthesized with novel 1,2-bis(isocyanate) ethoxyethane. Macromolecules 37, 3287-3291 (2004).

10 Furukawa, M., Fukumaru, T., Mitsui, Y. \& Kojio, K. Microphase-separated structure and mechanical properties of novel polyurethane elastomers prepared with ether based diisocyanate. Polymer 46, 10817-10822 (2005)

11 Kojio, K., Nakashima, S. \& Furukawa, M. Microphase-separated structure and mechanical properties of norbornane diisocyanate-based polyurethanes. Polymer 48, 997 1004 (2007).
12 Kojio, K., Kugumiya, S., Uchiba, Y., Nishino, Y. \& Furukawa, M. The microphaseseparated structure of polyurethane bulk and thin films. Polym. J. 41, 118-124 (2009).

13 Kojio, K., Nakamura, S. \& Furukawa, M. Effect of side methyl groups of polymer glycol on elongation-induced crystallization behavior of polyurethane elastomers. Polymer 45, 8147-8152 (2004)

14 Kojio, K., Nakamura, S. \& Furukawa, M. Effect of side groups of polymer glycol on microphase-separated structure and mechanical properties of polyurethane elastomers. J. Polym. Sci. Part B Polym. Phys. 46, 2054-2063 (2008).

15 Martin, D. J., Meijs, G. F. Renwick, G. M., Gunatillake, P. A. \& McCarthy, S. J. Effect of soft-segment ch2/o ratio on morphology and properties of a series of polyurethane elastomers. J. Appl. Polym. Sci. 60, 557-571 (1996).

16 Chen, W. P., Kenney, D. J., Frisch, K. C., Wong, S. W. \& Moore, R. Study of phase separation in polyurethane using paramagnetic labels effect of soft segment molecular weight, structure, and thermal history. J. Polym. Sci. Part B Polym. Phys. 29, 1513-1524 (1991).

17 Chen, W. P., Frisch, K. C., Kenney, D. J., Wong, S. W. \& Moore, R. Effect of soft segment molecular weight and 3-methyl side group on microstructural separation in polyurethane elastomers. J. Macromol. Sci. Pure Appl. Chem. 29, 567-587 (1992).

18 Martin, D. J., Meijs, G. F., Renwick, G. M., McCarthy, S. J. \& Gunatillake, P. A. The effect of average soft segment length on morphology and properties of a series of polyurethane elastomers. J. Appl. Polym. Sci. 62, 1377-1386 (1996).

19 Kojio, K., Nonaka, Y. \& Furukawa, M. Effect of the composition ratio of copolymerized poly(carbonate) glycol on the microphase-separated structures and mechanical properties of polyurethane elastomers. J. Polym. Sci. Part B Polym. Phys. 42, 4448-4458 (2004).

20 Kojio, K., Furukawa, M., Motokucho, S., Shimada, M. \& Sakai, M. Structure-mechanical property relationship for poly(carbonate urethane) elastomers with novel soft segments. Macromolecules 42, 8322-8327 (2009).

21 Masubuchi, T., Sakai, M., Kojio, K., Furukawa, M. \& Aoyagi, T. Structure and properties of aliphatic poly(carbonate) glycols with different methylene unit length e-J. Soft Mater. 3, 55-63 (2007).

22 Casetta, C., Girelli, D. \& Greco, A. Polycarbonate diols: a new way to synthesize high performance polyurethanes. Pitture e Vernici Europe 76, 9-16 (1994).

23 Furukawa, M. Property-structure relationships of polyurethane elastomers: improvement of hydrolytic stability and thermal stability. J. Appl. Polym. Sci Appl. Polym. Symp. 53, 61-76 (1994)

24 Kimura, I., Ishihara, H., Ono, H., Yoshihara, N., Nomura, S. \& Kawai, H. Morphology and deformation mechanism of segmented poly(urethaneureas) in relation to spherulitic crystalline textures. Macromolecules 7, 355-363 (1974).

25 Desper, C. R., Schneider, N. S., Jasinski, J. P. \& Lin, J. S. Deformation of microphase structures in segmented polyurethanes. Macromolecules 18, 2755-2761 (1985).

26 Blundell, D. J., Eeckhaut, G., Fuller, W., Mahendrasingam, A. \& Martin, C. Real time SAXS/stress-strain studies of thermoplastic polyurethanes at large strains. Polymer 43, 5197-5207 (2002).

27 Koerner, H., Kelley, J. J. \& Vaia, R. A. Transient microstructure of low hard segment thermoplastic polyurethane under uniaxial deformation. Macromolecules 41, 47094716 (2008).

28 Waletzko, R. S., Korley, L. T. J., Pate, B. D., Thomas, E. L. \& Hammond, P. T. Role of increased crystallinity in deformation-induced structure of segmented thermoplastic polyurethane elastomers with PEO and PEO-PPO-PEO soft segments and HDI hard segments. Macromolecules 42, 2041-2053 (2009).

29 Masunaga, H., Ogawa, H., Takano, T., Sasaki, S., Goto, S., Tanaka, T., Seike, T., Takahashi, S., Takeshita, K., Nariyama, N., Ohashi, H., Ohata, T., Furukawa, Y., Matsushita, T., Ishizawa, Y., Yagi, N., Takata, M., Kitamura, H., Sakurai, K., Tashiro, K., Takahara, A., Amamiya, Y., Horie, K., Takenaka, M., Kanaya, T., Jinnai, H., Okuda, H., Akiba, I., Takahashi, I., Yamamoto, K., Hikosaka, M., Sakurai, S., Shinohara, Y., Okada, A. \& Sugihara, Y. Multipurpose soft-material SAXS/WAXS/GISAXS beamline at SPring-8. Polym. J. 43, 471-477 (2011).

30 Hwang, K. K. S., Wu, G., Lin, S. B. \& Cooper, S. L. Synthesis and characterization of MDI-butanediol urethane model compounds. J. Polym. Sci. 22, 1677-1697 (1984).

31 Blackwell, J. \& Lee, C. D. Hard-segment polymorphism in MDI/diol-based polyurethane elastomers. J. Polym. Sci. Polym. Phys. 22, 759-772 (1984).

32 Imada, K., Miyakawa, T., Chatani, H., Tadokoro, H. \& Murahashi, S. Structural studies of polyethers, [-(CH2)mO-]n. III. Molecular and crystal structure of poly(tetrahydrofuran). Makromol. Chem. 83, 113-122 (1965). 